\title{
ARTERIOVENOUS FISTULA AFTER CEREBRAL VENOUS SINUS THROMBOSIS
}

\author{
LOW QIN JIAN ${ }^{1}$, CHEO SENG WEE ${ }^{2}$
}

\begin{abstract}
Thrombosis of the deep cerebral vein is a rare entity. Thrombosis of cerebral veins or dural sinus obstructs blood drainage from brain tissue, leading to cerebral parenchymal dysfunction and increased venous and capillary pressure with disruption of the blood-brain barrier. Many conditions are associated with cerebral venous sinus thrombosis. Prothrombotic conditions, malignancy, infection and head injury has been reported as risk factors for cerebral venous sinus thrombosis (CVST). Neuroimaging features of CVST include focal areas of oedema or venous infarction, hemorrhagic venous infarction, diffuse brain edema or rarely subarachnoid haemorrhage. Magnetic resonance imaging of the brain in combination with magnetic resonance venography is the most informative technique in demonstrating the presence of dural thrombus and cortical vein thrombosis.
\end{abstract}

Keywords : Arteriovenous fistula (AVF), cerebral venous sinus thrombosis (CVST).

Received: 03 September 2018

Accepted: 05 November 2018

DOI: https://doi.org/10.3329/bjmed.v30i1.39921

\section{Introduction}

Cerebral venous sinus thrombosis is a rare form of venous thromboembolism with an estimated annual incidence of $3-7$ per $1,000,000$ populations ${ }^{1}$. The development of dural arteriovenous fistula (AVF) after CVST is rare and have been reported for only several cases $^{2}$. Moreover, in all these cases, dural AVF was presented as the chronic complication of CVST, which developed several months or years later. We report a case of likely arteriovenous fistula (AVF) following cerebral venous sinus thrombosis with meningoencephalitis.

\section{Case Report}

A 51-years-old gentleman with no comorbid experienced fever and headache for 3 days' duration. He had been fasting for the last ten days during Ramadan. On arrival, his Glasgow Coma Scale was E1 V2 M5. Neurological examination revealed an increase tone at bilateral upper and lower limbs and generalized brisk reflexes with bilateral down-going plantar response. Brain computed tomography showed a linear hyperdensity along the posterior inter-hemispheric fissure and right transverse sinus.
MRV brain showed the presence of filling defect within the deep cerebral venous sinus involving the internal cerebral vein, vein of Galen and straight sinus. Cerebrospinal fluid (CSF) study showed an opening pressure of $30 \mathrm{~cm}$ water, turbid appearance, 50 cells $/ \mathrm{mm}^{3}$, predominantly $60 \%$ neutrophils, CSF protein raised at $4.1 \mathrm{~g} / \mathrm{L}, \mathrm{CSF}$ glucose $2.6 \mathrm{mmol} / \mathrm{L}$, $\mathrm{CSF} /$ serum glucose ratio of $46 \%$. CSF gram stain, latex agglutination, tuberculous polymerase chain reaction, acid fast bacilli, indian ink and cryptococcal antibody were all negative. Marais score was not suggestive for TB meningitis. There was no evidence of malignancy, head trauma, polycythemia. His antiphospholipid study was negative. He was treated for bacterial meningoencephalitis with deep cerebral vein thrombosis and completed 2 weeks of intravenous ceftriaxone $2 \mathrm{~g}$ twice daily and subcutaneous enoxaparin $1 \mathrm{mg} / \mathrm{kg}$ twice daily. Upon discharge, his GCS was full. He was given a total of 3 months of anticoagulation therapy for his provoked cerebral venous sinus thrombosis. He remained well during his follow-up at 1 year with no residual deficits.

1. Physician, Department Of Medicine, Hospital Sultanah Nora Ismail, Johor, MALAYSIA.

2. Physicina, Department of Medicine, Hospital Lahad Datu, Sabah, Malaysia.

Address of correspondence: Low Qin Jian, Physician, Department Of Medicine, Hospital Sultanah Nora Ismail, Johor, MALAYSIA. Email: peterlow4964@gmail.com

Bangladesh J Medicine 2019; 30 : 35-37 

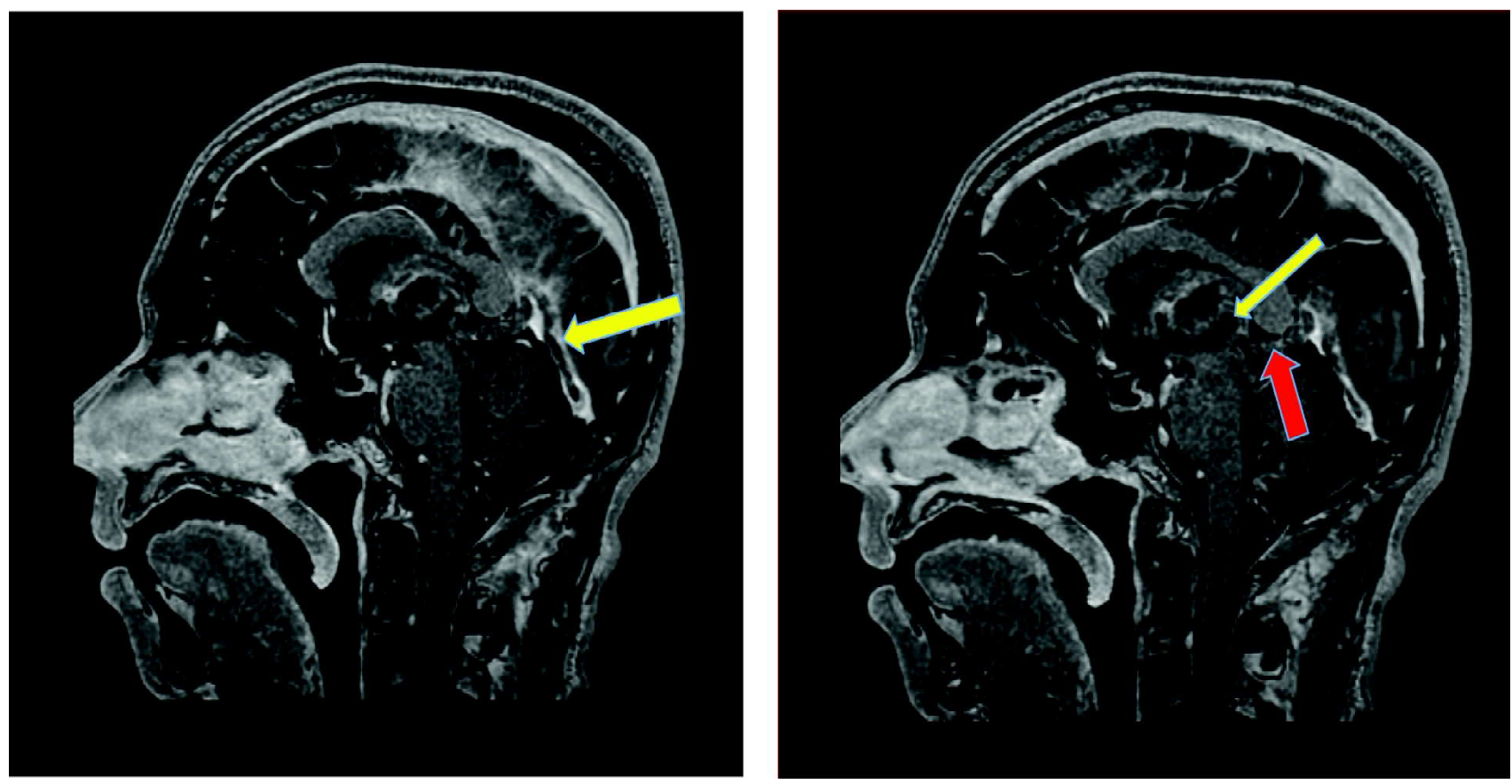

Figure 1 (Above, Left) : MRI brain T1-post contrast sequence- (sagittal view) - shows evidence of filling defect along the straight sinus (yellow arrow) suggestive of venous sinus thrombosis.

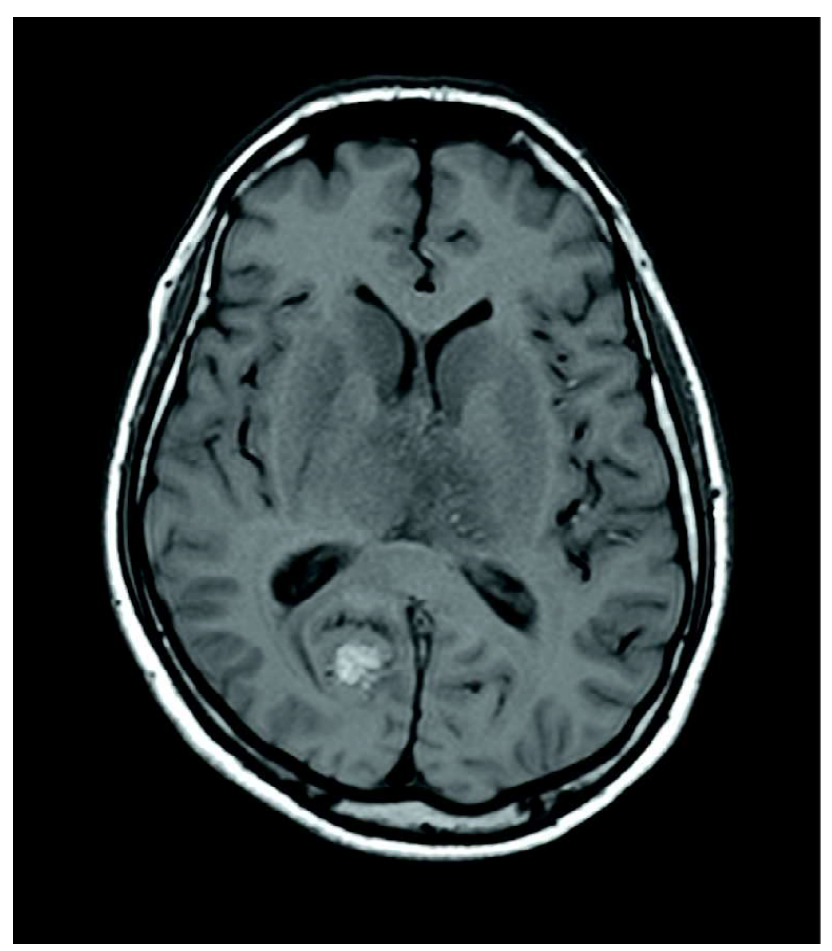

Figure 2 (Above, Right) : MRI brain T1-post contrast sequence-(sagittal view) - shows evidence of filling defect along the internal cerebral vein (yellow arrow) and vein of Galen (red arrow) suggestive of deep cerebral venous sinus thrombosis.

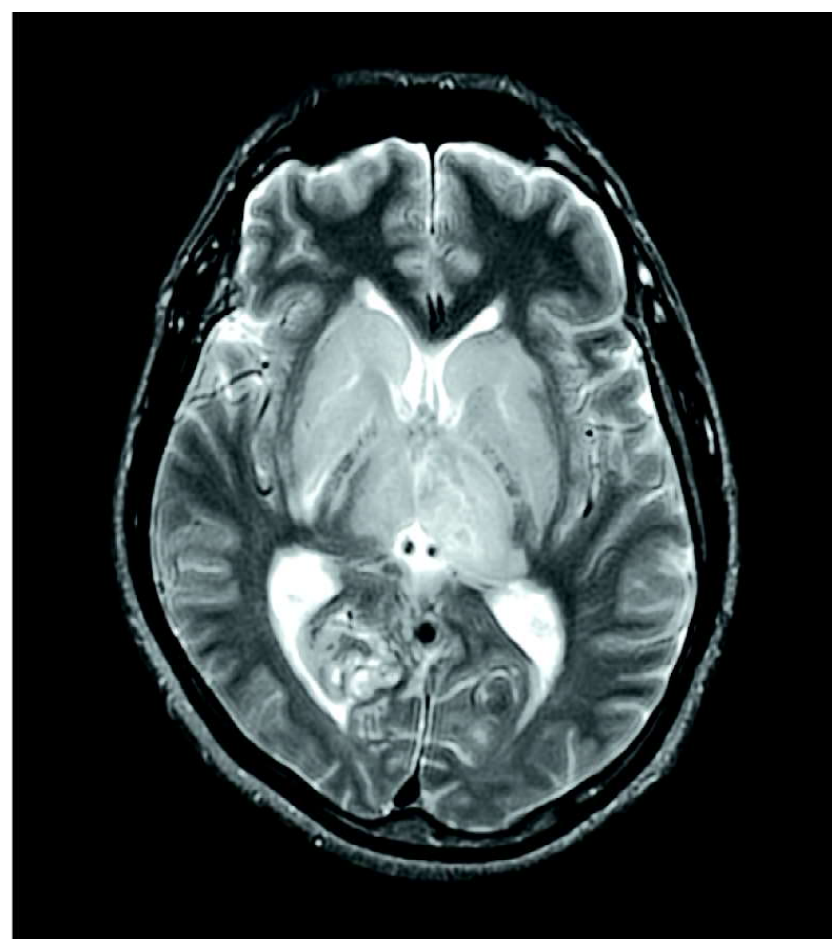

Figure 3 and 4 (Above, Left and Right): MRI brain (axial view) in T1-weighted sequence (a) and T2-weighted sequence (b) shows serpinginous structures at the right occipital lobe which suggestive of vascular malformations. 


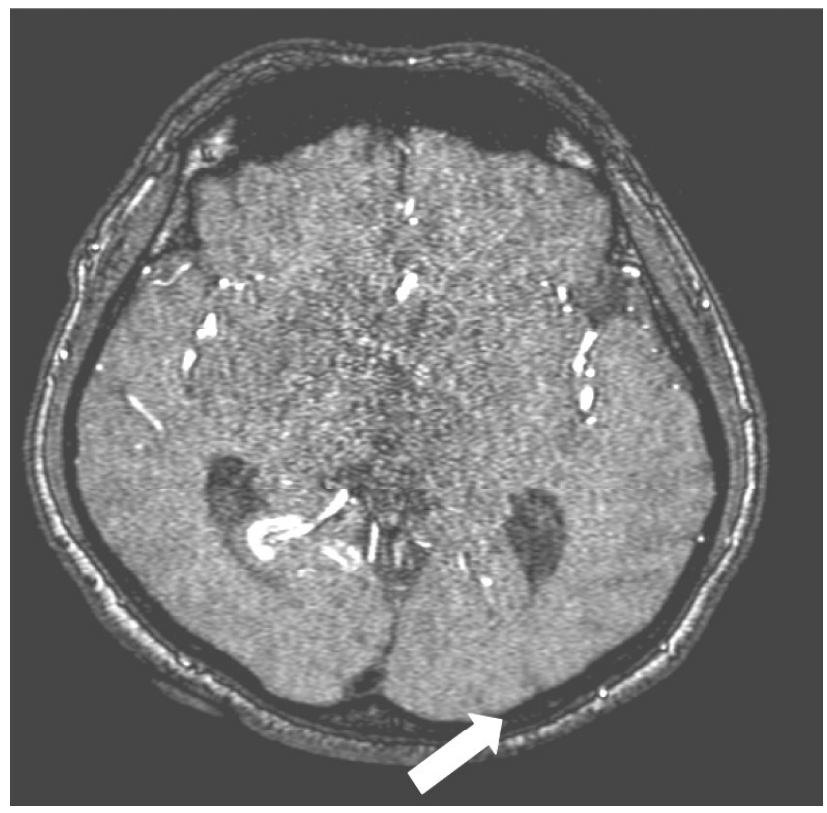

Fig.-5 (Above, left): MRI brain T1-post contrast sequence (axial view) shows evidence of filling defect along the internal cerebral vein (yellow arrow) suggestive of deep cerebral venous sinus thrombosis.

\section{Discussion}

Two hypotheses have been proposed for the pathogenesis of AVF. The first is based on the physiological arteriovenous shunts between the meningeal arterial networks and the dural venous sinuses. An increase in sinus and venous pressure, for example, by the obstruction of venous outflow by CVST, may open these channels to create dural AVFs. The second hypothesis suggests that venous hypertension induced by an obstruction to venous outflow may reduce cerebral perfusion and lead to ischaemia, followed by angiogenesis. The aberrant angiogenic activity of the dural blood vessels would then result in arteriovenous shunting. In both, CVST may be the primary event that caused the venous hypertension. Despite constant investigation about relationships between AVF and CVST, whether CVST is the cause or result of AVF remains elusive. In our case, it is difficult to decide AVF was primary or not without baseline cerebral angiography. Nearly $40 \%$ of patients with CVST would have seizures as the presenting feature and among patients with seizures.

\section{Conclusions}

We postulated that the vascular malformation is likely an arteriovenous fistula due to venous hypertension following the venous sinus thrombosis. The venous hypertension developed after venous thrombosis opens up the microvascular connections within the dura and

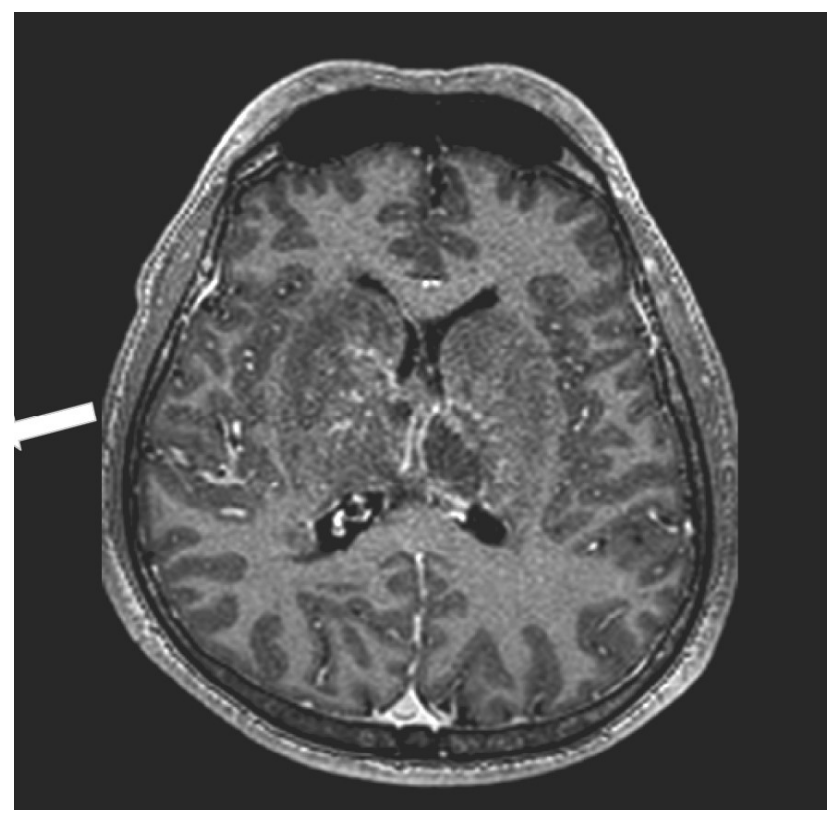

Fig.-6: (Above, right): MRI brain MRA TOF sequence (axial view) shows the serpinginous structures at the right occipital lobe being supplied by the right posterior cerebral artery (yellow arrow).

these channels become hypertrophied resulting in direct shunting between arteries and veins. This patient does not have a baseline brain computed tomography done previously.

\section{Conflict of interest}

The authors declare that they have no competing interests.

\section{Consent}

Written informed consent was obtained from the patient to publish the case. A copy of the written consent is available for review by the Chief Editor of this journal.

\section{References}

1. Tam J. Thrombosis of the cerebral veins and sinus. $\mathrm{N}$ Engl J Med 2005;352:1791-8.

2. Morales H, Jones BV, Leach JL, et al. Documented development of a dural arteriovenous fistula in an infant subsequent to sinus thrombosis: case report and review of the literature. Neuroradiology 2010; 52:225-9.

3. Micieli JA, Derkatch S, Pereira VM, et al. Development of dural arteriovenous fistulas after cerebral venous sinus thrombosis. J Neuroophthalmol 2016;36:53-7.

4. Nishijima M, Takaku A, Endo S, et al. Etiological evaluation of dural arteriovenous malformations of the lateral and sigmoid sinuses based on histopathological examinations. J Neurosurg 1992;76:600-6. 